\title{
Validation of an Adaptive Transfer Function Method to Estimate the Aortic Pressure Waveform
}

\author{
Yang Yao, Lisheng Xu, Yingxian Sun, Qiang Fu, Shuran Zhou, Dianning He, Yahui Zhang, Liang Guo, \\ and Dingchang Zheng
}

\begin{abstract}
Aortic pulse wave reflects cardiovascular status, but unlike the peripheral pulse wave, is difficult to be measured reliably using non-invasive techniques. Thus, the estimation of aortic pulse wave from peripheral ones is of great significance. This study proposed an adaptive transfer function (ATF) method to estimate the aortic pulse wave from the brachial pulse wave.

Aortic and brachial pulse waves were derived from 26 patients who underwent cardiac catheterization. Generalized transfer functions (GTF) were derived based on the autoregressive exogenous model. Then the GTF was adapted by its peak resonance frequency. And the optional peak resonance frequency for an individual was determined by regression formulas using brachial systolic blood pressure. The method was validated using the Leave-One-Out Cross Validation method.

Compared with previous studies, the ATF method showed better performance in estimating the aortic pulse wave and predicting the feature parameters. The prediction error of the
\end{abstract}

This work was supported by the National Natural Science Foundation of China (Nos. 61374015 and 61202258), and the Fundamental Research Funds for the Central Universities (Nos. N130404016 and N110219001).

Yang Yao is with the Sino-Dutch Biomedical and Information Engineering School, Northeastern University, Shenyang City, Liaoning Province, 110819 China.(e-mail: yy511721925@163.com)

Lisheng $\mathrm{Xu}$ is with the Sino-Dutch Biomedical and Information Engineering School, Northeastern University, Shenyang City, Liaoning Province, 110819 China, and is also with the Key Laboratory of Medical Image Computing (Northeastern University), Ministry of Education, China. (e-mail: xuls@bmie.neu.edu.cn)

Yingxian Sun is with the department of cardiology, the First Hospital of China Medical University, Shenyang City, Liaoning Province, 110001 China, and is also a part-time professor in the Sino-Dutch Biomedical and Information Engineering School, Northeastern University, Shenyang City, Liaoning Province, 110819 China. (e-mail: sunyingxian@ medmail.com.cn)

Qiang Fu is with the Sino-Dutch Biomedical and Information Engineering School, Northeastern University, Shenyang City, Liaoning Province, 110819 China.(e-mail:1244813162@qq.com)

Shuran Zhou is with the Sino-Dutch Biomedical and Information Engineering School, Northeastern University, Shenyang City, Liaoning Province, 110819 China.(e-mail: $514909166 @$ qq.com)

Dianning $\mathrm{He}$ is with the Sino-Dutch Biomedical and Information Engineering School, Northeastern University, Shenyang City, Liaoning Province, 110819 China.(e-mail: 159258711 @qq.com)

Yahui Zhang is with the Sino-Dutch Biomedical and Information Engineering School, Northeastern University, Shenyang City, Liaoning Province, 110819 China.(e-mail: $1171518067 @$ qq.com)

Liang Guo is with the department of cardiology, the First Hospital of China Medical University, Shenyang City, Liaoning Province, 110001 China.(e-mail: doctorgl@126.com)

Dingchang Zheng is with the First Health \& Wellbeing Academy, Faculty of Medical Science, Anglia Ruskin University, Chelmsford, UK, CM1 1SQ. (e-mail: dingchang.zheng@newcastle.ac.uk)

Lisheng Xu and Yingxian Sun are co-corresponding authors. aortic systolic blood pressure and pulse pressure were 0.2 \pm 3.1 $\mathrm{mmHg}$ and $-0.9 \pm 3.1 \mathrm{mmHg}$, respectively. The percentage errors of augmentation index, percentage notch amplitude and ejection duration were $-2.1 \pm 32.7 \%, \quad 12.4 \pm 9.2 \%$, and $-2.4 \pm 3.3 \%$, respectively.

\section{Index Terms - aortic pulse wave, adaptive transfer function}

\section{INTRODUCTION}

CENTRAL aortic pulse wave is more informative about cardiovascular status and thus provides greater clinical value over peripheral pulse waves [1-4]. However, it cannot be readily obtained accurately and reliably using a non-invasive technique, thus its application in clinical practice is limited. On the other hand, pulse waves at peripheral sites are non-invasively available. Thus, it is natural to consider estimating the aortic pulse wave using peripheral pulse waves.

One way is to use the carotid pulse wave as a surrogate [5-6]. However, this method is limited by the inconvenient measurement of carotid pulse wave. In addition, despite the similarity of the aortic and carotid pulse wave, the amplitude of the augmented pressure wave in the ascending aorta is much higher than that in the carotid artery [7-8], which affects the calculation precision of some cardiovascular parameters like the augmentation index (AI).

Another way is to simulate the relationship between central and peripheral pulse waves. The most commonly used is the generalized transfer function (GTF) method. Chen et al. [9] evaluated the validity of using an inverse aortic-radial model to estimate the aortic pulse wave. Fetics [10] extended Chen's study by presenting a comparison between the direct radial-aortic model and the inverse aortic-radial model. Pauca [11] evaluated a commercially available device (SphygmoCor, AtCor, Australia) which employed the GTF method to estimate the aortic pulse wave. However, the GTF method does not account for the inter-subject or intra-subject variability of the transfer function (TF). Stok [12] employed two second order filters for the estimation and adapted its resonance frequency for each individual. However, these two filters did not fit the true GTF well enough especially at frequency bands near $0 \mathrm{~Hz}$. Subject-specific methods [13-16] were proposed in recent years, but were unfortunately rarely used in clinical practice. This paper aims to propose a novel method using adaptive transfer function (ATF) to estimate the aortic pulse wave. 


\section{METHODS}

For a higher accuracy of modeling, a pre-processing procedure including denoising, detrending, and normalization should be applied to both the aortic and brachial pulse waves. However, the detrending and normalization procedures eliminate the trend (fluctuation of blood pressure) of the pulse waves. Thus, two GTFs $H_{g 1}$ and $H_{g 2}$ were built, with $H_{g 1}$ created from the undetrended data and $H_{g 2}$ from the detrended data. When estimating the aortic from the brachial pulse wave, the two GTFs $H_{g 1}$ and $H_{g 2}$ were adaptively adjusted, deriving two ATFs $H_{a 1}$ and $H_{a 2}$, respectively. Then, the normalized aortic pulse wave (detrended) and the trend were obtained by applying $H_{a 2}$ and $H_{a 1}$ to the brachial pulse wave, respectively. Then, the desired aortic pulse wave was derived by calibrating the normalized aortic pulse wave to the trend.

In detail, in the modeling procedure, two TFs named $H_{i 1}$ and $H_{i 2}$ were firstly created from each data set. As shown in Fig. 1 (a), $H_{i 1}$ was derived directly from the denoised data, and $H_{i 2}$ was derived using the normalized data. Next, all the derived $H_{i 1}$ and $H_{i 2}$ were averaged to create GTFs $H_{g 1}$ and $H_{g 2}$, respectively. Finally, the two generalized were adaptively adjusted to derive ATFs $H_{a 2}$ and $H_{a 1}$. As shown in Fig. 1 (b), when estimating the aortic pulse wave, the brachial pulse wave with trend and the one detrended were obtained in the same way as in the modeling procedure. And the desired aortic pulse wave was reconstructed by applying $H_{a 2}$ to the brachial pulse wave (with no trend) and calibrated to the foot and mean of the $H_{a 1}$-reconstructed aortic pulse wave (with trend). The calibration procedure using the $H_{a 1}$-estimated aortic pulse wave is the inverse of the normalization procedure.

\section{A. Data Collection}

Twenty-six patients who underwent cardiac catheterization at the First Hospital of China Medical University were enrolled for this study. There were 10 men and 16 women at the ages ranging from 29 to 78 years old (mean $\pm \mathrm{SD}, 57 \pm 12$ years). Informed consents were obtained in all subjects. This study was approved by the Sino-Dutch Biomedical and Information Engineering School, Northeastern University, CN. Detailed clinical characteristics of the subjects are given in TABLE I. To better test the existence of a stable aorta-brachial relationship and the accuracy of the TF-based approach, invasive data measured using pressure wires were used. The aortic and brachial pulse waves were recorded by two micro-manometers, which were placed in the ascending aorta and the brachial artery, respectively. As the frequency components of both central and peripheral arterial pulse waves are under $30 \mathrm{~Hz}$ [17], and higher frequency sampling frequency brings in more high frequency components which affects the accuracy of modeling, the data were digitized at $100 \mathrm{~Hz}$.

\section{B. Data Pre-processing}

In this part, the pre-filtering and data segmentation procedures were employed for the derivation of both $H_{i 1}$ and $H_{i 2}$. While the de-trending and normalization procedures were only used for the derivation of $H_{i 2}$ as shown in Fig. 1.

In order to eliminate the high frequency noise, the data were smoothed by a 4th order Butterworth low-pass filter. As the frequency components of the pulse wave are mainly less than $10 \mathrm{~Hz}$ [17], the cut-off frequency of the filter was set to $15 \mathrm{~Hz}$.

As blood pressure (BP) of an individual fluctuates, low-frequency trend is contained in the pulse wave signal. This low-frequency trend, together with the low-frequency drift caused by respiration, motion artifact and neural regulation, influences the modeling accuracy by reducing the weights of

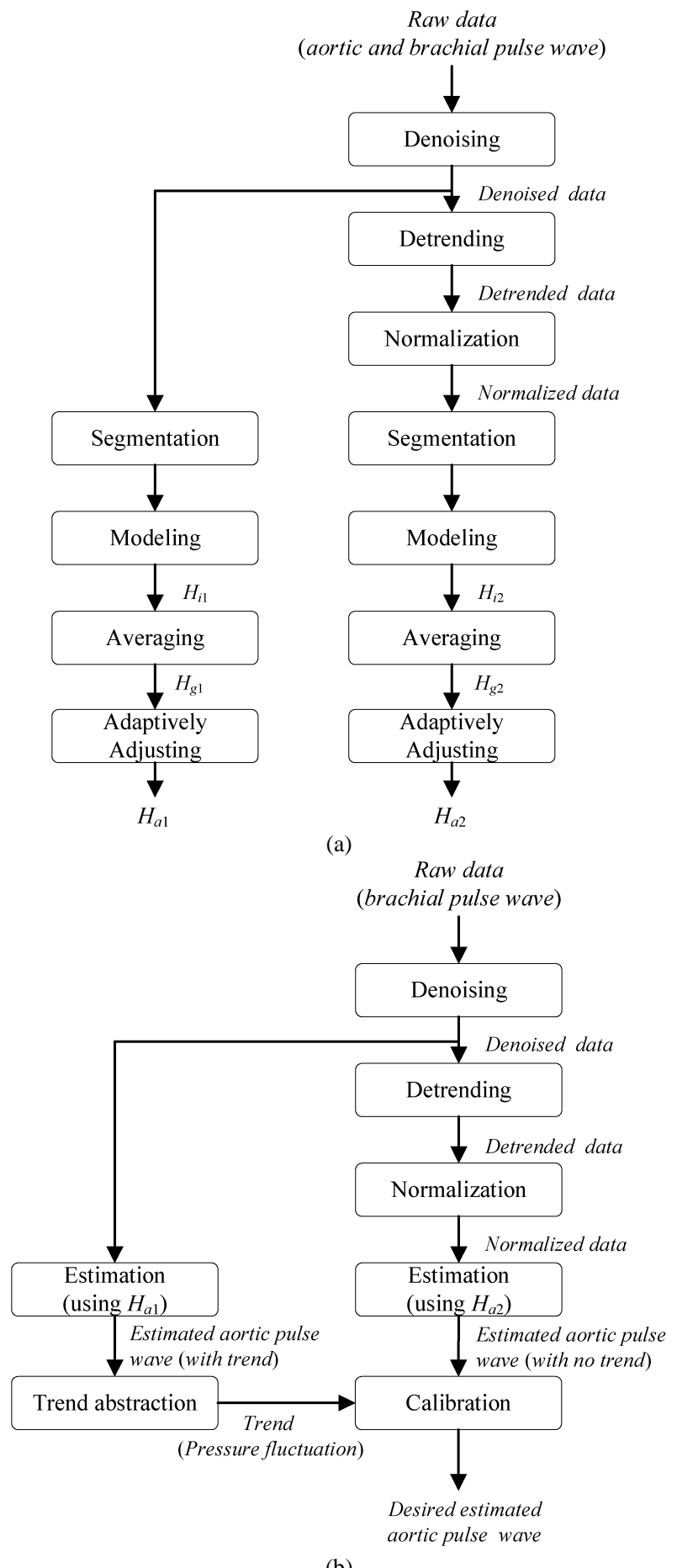

(b)

Fig. 1. Steps of the modeling and estimation procedures: (a) Modeling procedure in which $H_{a 1}$ and $H_{a 2}$ were built based on aortic and brachial pulse waves; (b) Estimation procedure in which aortic pulse wave was estimated by applying $H_{a 1}$ and $H_{a 2}$ to brachial pulse wave. 
TABLE I

SUBJECT CHARACTERISTICS AND PHYSIOLOGIC INFORMATION

\begin{tabular}{|c|c|c|c|c|c|c|c|c|c|c|c|}
\hline No. & Sex & Age, y & Height, $\mathrm{cm}$ & Weight, kg & $\mathrm{HR}, \mathrm{bpm}$ & $\mathrm{SBP}_{\mathrm{ao}}$ & $\mathrm{SBP}_{\mathrm{br}}$ & $\triangle \mathrm{SBP}$ & $\mathrm{DBP}_{\text {aо }}$ & $\mathrm{DBP}_{\mathrm{br}}$ & $\triangle \mathrm{DBP}$ \\
\hline 1 & $\mathrm{M}$ & 63 & 173 & 79 & 62 & 132 & 141 & 9 & 66 & 68 & 2 \\
\hline 2 & F & 45 & 167 & 65 & 75 & 149 & 155 & 6 & 89 & 91 & 2 \\
\hline 4 & F & 60 & 160 & 69 & 86 & 165 & 175 & 10 & 90 & 95 & 5 \\
\hline 5 & M & 59 & 169 & 72 & 66 & 136 & 137 & 1 & 78 & 78 & 0 \\
\hline 6 & F & 72 & 165 & 80 & 69 & 178 & 188 & 10 & 76 & 82 & 6 \\
\hline 8 & F & 57 & 165 & 65 & 73 & 123 & 148 & 25 & 65 & 68 & 3 \\
\hline 9 & F & 58 & 159 & 53 & 63 & 141 & 148 & 7 & 75 & 75 & 0 \\
\hline 10 & F & 61 & 150 & 57 & 69 & 181 & 190 & 9 & 75 & 75 & 0 \\
\hline 11 & F & 36 & 168 & 70 & 86 & 105 & 111 & 6 & 69 & 69 & 0 \\
\hline 12 & F & 69 & 160 & 72 & 77 & 178 & 182 & 4 & 75 & 81 & 6 \\
\hline 16 & F & 59 & 158 & 60 & 75 & 145 & 146 & 1 & 76 & 75 & -1 \\
\hline 17 & M & 60 & 170 & 75 & 58 & 123 & 129 & 6 & 64 & 64 & 0 \\
\hline 18 & F & 56 & 160 & 60 & 92 & 130 & 143 & 13 & 74 & 72 & -2 \\
\hline 19 & F & 61 & 156 & 49 & 81 & 138 & 140 & 2 & 73 & 69 & -4 \\
\hline 20 & F & 60 & 164 & 75 & 100 & 184 & 194 & 10 & 93 & 98 & 5 \\
\hline 21 & $\mathrm{M}$ & 35 & 173 & 65 & 65 & 111 & 124 & 13 & 78 & 79 & 1 \\
\hline 22 & F & 63 & 158 & 64 & 74 & 149 & 159 & 10 & 73 & 77 & 4 \\
\hline 23 & $\mathrm{M}$ & 66 & 169 & 66 & 86 & 143 & 158 & 15 & 69 & 70 & 1 \\
\hline 24 & $\mathrm{M}$ & 76 & 170 & 55 & 62 & 160 & 168 & 8 & 85 & 83 & -2 \\
\hline 25 & $\mathrm{M}$ & 44 & 173 & 85 & 67 & 141 & 143 & 2 & 84 & 82 & -2 \\
\hline
\end{tabular}

HR, heart rate; SBP, systolic blood pressure; DBP, diastolic blood pressure; $\mathrm{SBP}_{\mathrm{ao}}$ and $\mathrm{SBP}_{\mathrm{br}}$ are the measured aortic and brachial SBP, respectively; DBP and $\mathrm{DBP}_{\mathrm{br}}$, are the measured aortic and brachial DBP, respectively; $\Delta$, pressure difference between the brachial artery and the aorta; SD, standard derivation.

other frequency components during parameter calculation using least squares method. The low-frequency trend was removed by applying a $7^{\text {th }}$ order wavelet decomposition to the pulse signal, eliminating the approximation coefficients [18]. To equate the mean and diastolic pressures in brachial and aortic pulse waves, and also, to further eliminate the influence of the beat-to-beat pulse wave change on the calculation of model parameters, the pulse wave was normalized in amplitude, with the mean to zero and the foot to -1 .

Data length significantly influences the accuracy of modeling, for too long a series of data may cause information-saturation, whereas too short a series of data may poorly describe the system dynamics. Thus, each data set was divided into 1-5 groups of 2000 sampling points each. Several TFs were derived from the data segments and then averaged.

\section{Derivation of the Generalized Transfer Function}

An autoregressive exogenous (ARX) model [19] was used in this study, which is expressed as

$$
\begin{aligned}
y(t)= & -a_{1} y(t-1)-\cdots-a_{n_{a}} y\left(t-n_{a}\right)+b_{1} u(t-k)+\cdots \\
& +b_{n_{b}} u\left(t-k-n_{b}+1\right)+\varepsilon(t)
\end{aligned}
$$

where $k$ is the time delay between input $u$ and output $y, \varepsilon$ is the residual error between the estimated and actual output. $n_{a}$ and $n_{b}$ are the number of $a_{i}$ and $b_{j}$ coefficients of the model, respectively. In this paper, they were both set equal to the model order. Time delay $k$ was set to zero and the aortic and brachial pulse waves were shifted together for convenience.

The parameters $a_{i}$ and $b_{j}$ were determined by minimizing the $\varepsilon(t)$ using the Least-Squares Estimation (LSE) method [19]. As mentioned above, the data series recorded from each subject was divided into several segments, thus the parameters were calculated from all segments and then averaged.

\section{Model Order Determination}

Model order is a determinant of the performance of the system. Too high a model order may bring in extra interference, whereas too low an order may be not enough for describing a certain system. In this paper, Akaike's Final Prediction Error $(F P E)$ [19] was used as a criterion to choose the appropriate order for the model. The FPE $\varepsilon_{F}$ is calculated as:

$$
\varepsilon_{F}=\frac{1+\left(n_{a}+n_{b}\right) / N}{1-\left(n_{a}+n_{b}\right) / N} L
$$

in which $n_{a}$ and $n_{b}$ are the numbers of coefficients $a_{i}$ and $b_{j}$, respectively. $N$ is the length of data. $L$ is the loss function:

$$
L=\frac{1}{N} \sum \varepsilon^{2}(t)
$$


The FPE derived from the $H_{i 2}$ based on different orders of 26 subjects are shown in Fig. 2. The FPE shows no evident change when model order ranges from 10 to 30 , whereas comparatively larger FPE is shown in small model orders (especially 4 and 5). Considering this, along with the fact that too high a model order may bring in high-frequency noise, the order was set to 10 .

\section{E. Derivation of the Adaptive Transfer Function}

Several parameters of the brachial pulse wave as well as features of each individual transfer function derived by the ARX method were calculated. The calculated parameters of the brachial pulse wave include systolic (SBP), diastolic (DBP), pulse (PP) and mean (MBP) BPs, heart rate (HR), cardiac output (CO), stroke volume (SV) and form factor (FF). CO and SV were calculated using the pressure-recording analytical method which is solely based on the peripheral pulse wave and does not require any prior calibration [20-22]. As suggested [20-21], the sampling rate of the brachial pulse wave was increased to $1000 \mathrm{~Hz}$ by interpolation for a better accuracy in the calculation of CO and SV. The key features of the transfer function include magnitude at $0 \mathrm{~Hz}$ and both magnitude and frequency of the first peak. The relationship between the parameters of the brachial pulse wave and the features of the transfer function is calculated. SBP is shown to be significantly associated with the peak resonance frequency of both the undetrended TF and the detrended TF $(r=0.59, \mathrm{p}<0.01$ and $\mathrm{r}=0.63, \mathrm{p}<0.001$, respectively). A significant correlation between MBP and the peak resonance frequency of both the undetrended TF and the detrended TF $(r=0.59, \mathrm{p}<0.01$ and $\mathrm{r}=0.57, \mathrm{p}<0.01$, respectively) was also found. However, compared with MBP, SBP correlates better with the detrended TF. In addition, brachial SBP is directly available using cuff method. Thus, the generalized transfer function was adaptively adjusted by shifting the peak resonance frequency according to the SBP of a specific individual. The regression formulas used

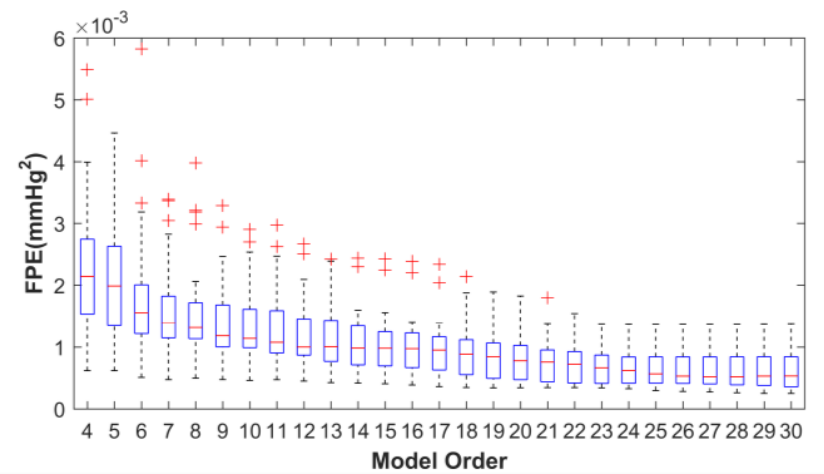

Fig. 2. Boxplot of FPE among subjects, grouped by model order. Each box is the FPE of different subjects with a certain model order.

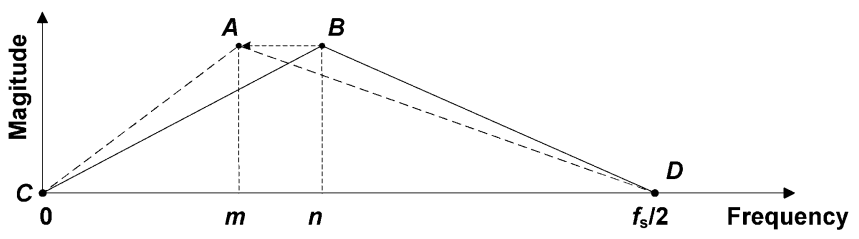

Fig. 3. Diagram of adaptively adjusting the GTF to the desired ATF. The solid line indicates the GTF and the dotted line indicates the desired ATF. $A$ and $B$ indicate the peaks of the desired ATF and the GTF, respectively. $m$ and $n$ are the peak resonance frequencies of the desired ATF and the GTF, respectively. to predict peak resonance frequencies from SBP $P_{\mathrm{s}}$ are:

$$
\begin{aligned}
& F_{\mathrm{p} 1}=\alpha^{*} P_{\mathrm{s}}+\tau_{1} \\
& F_{\mathrm{p} 2}=\beta^{*} P_{\mathrm{s}}+\tau_{2}
\end{aligned}
$$

where $F_{\mathrm{p} 1}$ and $F_{\mathrm{p} 2}$ are the target peak resonance frequencies of the undetrended TF and the detrended TF, respectively. $\alpha$ and $\beta$ are $0.012 \mathrm{~Hz} / \mathrm{mmHg}$ and $0.038 \mathrm{~Hz} / \mathrm{mmHg}$, respectively. $\tau_{1}$ and $\tau_{2}$ are $2 \mathrm{~Hz}$ and $-1.7 \mathrm{~Hz}$, respectively.

As shown in Fig. 3, solid line indicates the GTF, while dotted line indicates the desired ATF. $A$ and $B$ indicate the peaks of the desired ATF and the GTF, respectively. $m$ and $n$ are the peak resonance frequencies of the desired ATF and the GTF, respectively. To shift $n$ to $m$ ( $B$ to $A$ ), $C-B$ segment was compressed on frequency-axis yielding $C$ - $A$ segment, and similarly, $B-D$ segment was stretched on frequency-axis yielding $A-D$ segment.

\section{F. Prediction Evaluation}

To further evaluate the predictive performance of the proposed method, differences between the estimated and measured aortic pulse waves were evaluated. Several cardiovascular parameters were extracted from both the estimated and measured aortic pulse waves and then were compared. As shown in Fig. 4, SBP and DBP are defined as the amplitude of the peak and foot of the pulse wave, respectively; pulse pressure (PP) is defined as the difference between the SBP and DBP; Ejection duration (ED) is defined as the difference between the foot and the notch point in time; Augmentation index (AI) is calculated as ratio of the late systolic boost (amplitude difference between the peak and the inflection point) in the aortic pressure wave and PP [17]. Percentage notch amplitude $\left(p c t P_{n}\right)$, defined as the difference between the notch point and foot in amplitude divided by PP, was also included in performance evaluation.

When obtaining parameters from the pulse waves, $100 \mathrm{~Hz}$ digitization rate is far from sufficient, especially in determining the inflection point (which locates at the sharp upstroke of systolic duration). Thus, in order to obtain comparatively more accurate parameters, the measured and estimated aortic pulse waves were both interpolated by a factor of 10 before parameter calculation. The pulse waves were averaged using the ensemble average method. Feature parameters were then calculated from the average pulse wave. Prediction errors were calculated as the difference between the estimated and measured parameters.

In order to avoid over-fitting, and give a better insight on how well the model will generalize outside the dataset,

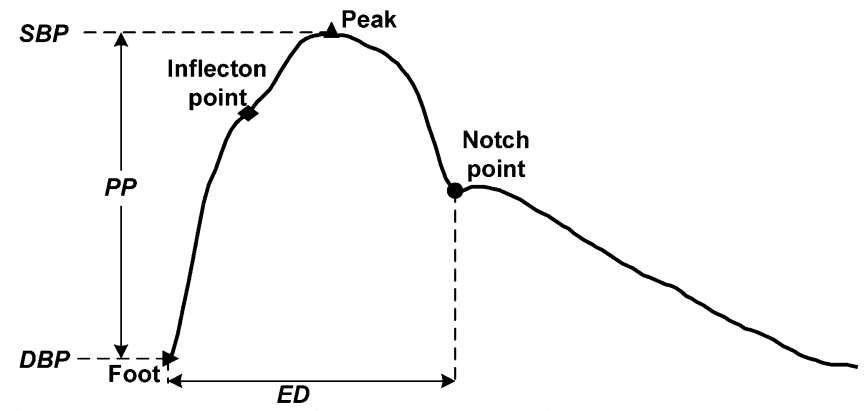

Fig. 4. Feature points and feature parameters of the aortic pulse wave. The inflection point is identified by the second peak of the second derivative of the aortic pulse wave. 
Leave-One-Out Cross-Validation (LOOCV) method was used in the validation of the proposed method.

The performance of this proposed method was compared with those of previous works by Chen [9], Fetics [11], and Im [23], and also with the N-Point Moving Average (NPMA) method, which was proposed by Williams [24] to estimate the central BPs from the radial pulse wave. This method was validated by Shih [25] to predict the aortic BP from the brachial pulse wave. The window width of the NPMA filter used in this paper is $f_{s} / 6$ (where $f s$ is the sampling rate of the pulse signal) as was used in Shih's study [25].

\section{RESULTS}

\section{A. Characteristics of transfer functions}

Fig. 5 shows the two GTFs $H_{g 1}$ and $H_{g 2}$. Increasing variations of $H_{g 1}$ and $H_{g 2}$ are obviously shown with frequency ranging from $0 \mathrm{~Hz}$ to the peak resonance frequency. The spectra of $H_{g 1}$ and $H_{g 2}$ show no obvious difference from 0 to $4 \mathrm{~Hz}$ except that

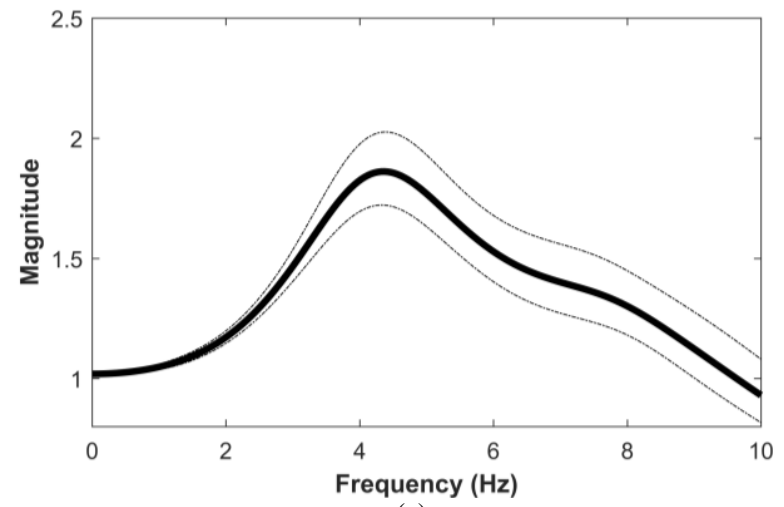

(a)

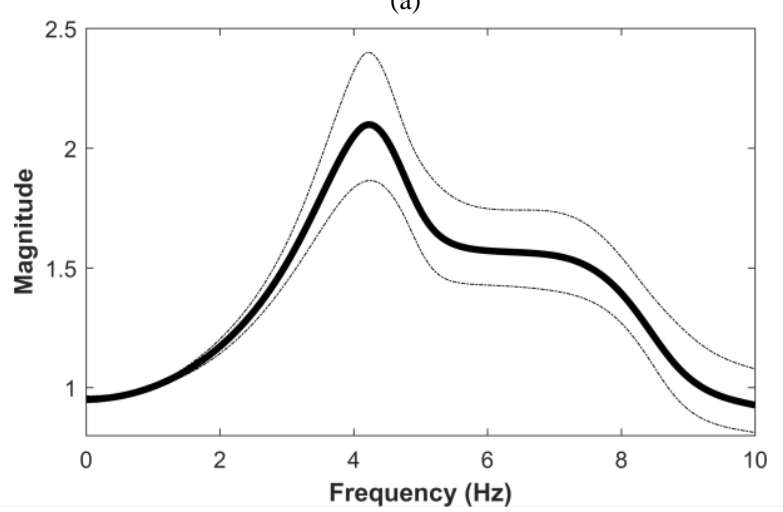

(b)

Fig. 5. Frequency response of two GTFs: (1) $H_{g 1}$; (2) $H_{g 2}$. (the area between the dash-dot lines is the $95 \%$ confidence interval)

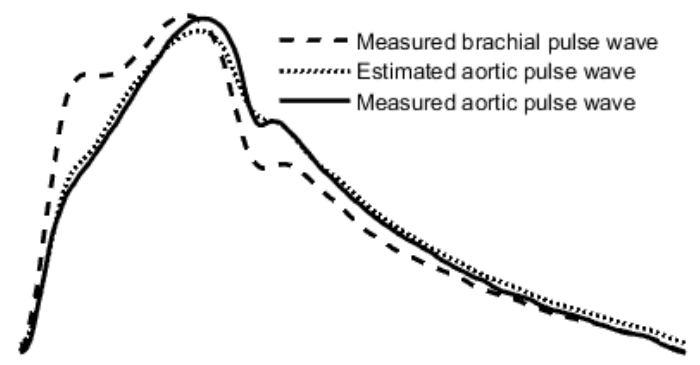

Fig. 6. Comparison among the measured brachial pulse waveform (bold dotted line), the measured aortic (bold solid line) and the estimated aortic (thin solid line with circle marker) pulse waves. the amplitude at $0 \mathrm{~Hz}$ is greater than 1 for $H_{g 1}$, and less than 1 for $H_{g 2}$. The location and the amplitude of the spectra peaks are similar, with the peak amplitude of $H_{g 1} 1.86$ at $4.7 \mathrm{~Hz}$ and $H_{g 2}$ 2.09 at $4.4 \mathrm{~Hz}$. Another difference between $H_{g 1}$ and $H_{g 2}$ is that obvious inflection points are shown at $6-8 \mathrm{~Hz}$ in the spectral plot of $H_{g 2}$, but not $H_{g 1}$.

\section{B. Evaluation of transfer functions}

Fig. 6 shows an example of the comparison among the measured brachial, measured aortic and estimated aortic pulse waves. The estimated aortic pulse wave is similar to the measured aortic pulse wave. While, the brachial pulse wave shows obvious difference against both the measured and estimated aortic pulse waves.

Fig. 7-8 show the Bland-Altman analysis of the measured and predicted aortic SBP and PP as well as of the measured brachial and aortic SBP and PP. The prediction error of SBP and $\mathrm{PP}$ are $0.2 \pm 3.1 \mathrm{mmHg}$ and $-0.9 \pm 3.1 \mathrm{mmHg}$, respectively. Whereas, the difference between brachial and aortic SBP is $7.5 \pm 5.4 \mathrm{mmHg}$ and the difference between brachial and aortic $\mathrm{PP}$ is $6.2 \pm 5.5 \mathrm{mmHg}$. The Bland-Altman analysis in Fig. 9 shows that the prediction error of $\mathrm{AI}$ ranges from $-29.3 \%$ to $16.1 \%$, whereas $\mathrm{AI}$ ranges from about $16.2 \%$ to $55.5 \%$. The percentage error of AI is $-2.1 \pm 32.7 \%$. Fig. 10 shows the comparison between the predicted and the measured $p c t P_{n}$. The percentage error of the $p c t P_{n}$ is $12.4 \pm 9.2 \%$. The range of the $p c t P_{n}$ error is $[-2.7,17.6] \%$, whereas the average of the predicted and the measured $p c t P_{n}$ ranges from $53.8 \%$ to $79.3 \%$. Fig. 11 shows comparison between the predicted and measured $\mathrm{ED}$, with a percentage error of $-2.4 \pm 3.3 \%$.

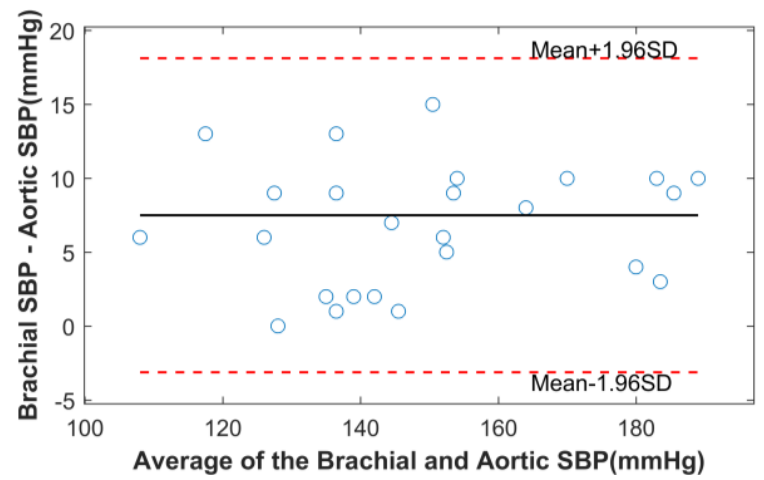

(a)

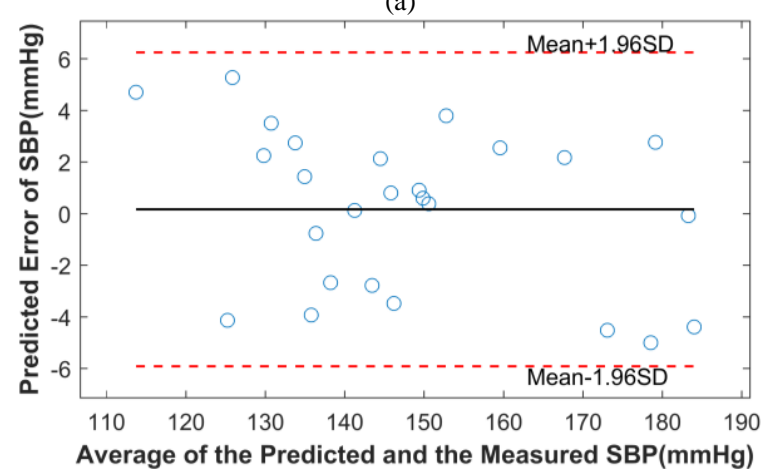

(b)

Fig. 7. Performance of the ATF method in estimating aortic SBP: (a) Comparison of the aortic SBP predicted using ATF method and the measured aortic SBP; (b) Comparison between the brachial and aortic SBP. The dashed line shows the mean difference; the dotted lines show the extent of 1.96 standard derivations (SD) from the mean. 


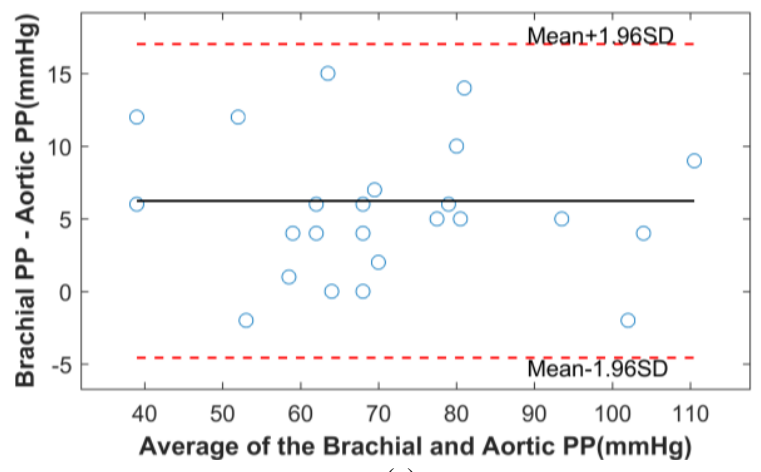

(a)

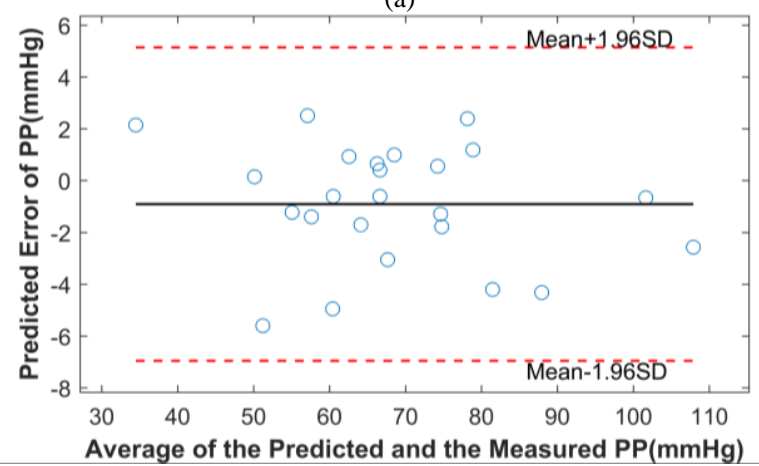

(b)

Fig. 8. Performance of the ATF method in estimating aortic PP: (a) Comparison of the aortic PP predicted using ATF method and the measured aortic PP; (b) Comparison between the brachial and aortic PP.

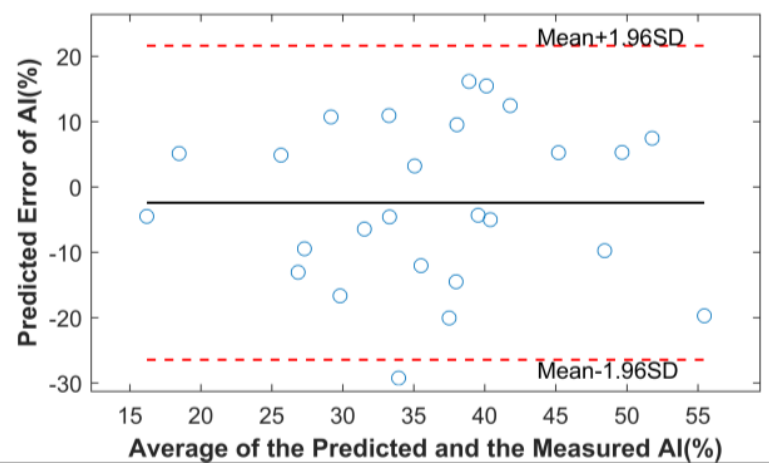

Fig. 9. Bland-Altman comparison between the AI predicted using ATF method and the measured AI.

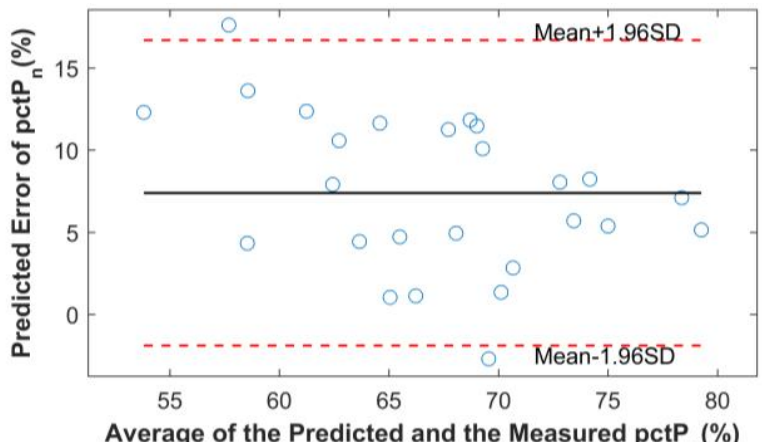

Fig. 10. Bland-Altman comparison between the $p c t P_{n}$ predicted using ATF method and the measured one.

TABLE II summaries the performance of the ATF method compared with three previous studies (by Chen [9], Fetics [6], and Im [23]) and the NPMA method [25]. The estimation performance of the proposed method in SBP is similar with Chen's, Fetics' and the NPMA method, and is better than Im's. The proposed method shows similar performance with Chen's

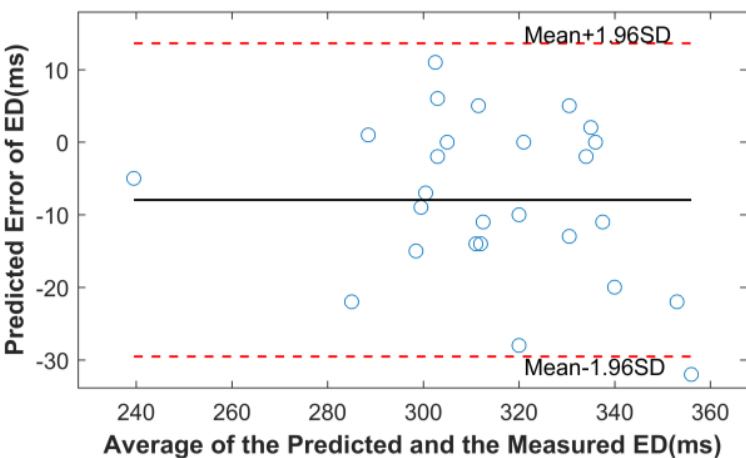

Fig. 11. Bland-Altman comparison between the ED predicted using ATF method and the measured ED.

and the NPMA method, but much better performance over Im's. The performance of AI calculation is much better than Chen's and Fetics', and is similar with Im's. And the performance of ED calculation is also much better than Fetic's [11].

\section{DISCUSSION AND CONCLUSION}

This study proposed an ATF method and assessed its feasibility of in estimating the aortic pulse wave from the brachial pulse wave.

For both $H_{g 1}$ and $H_{g 2}$, inter-patient variability is low in lower frequency band, but increases from $0 \mathrm{~Hz}$ to the peak resonance frequency and then stays stable. However, as the amplitude of the GTFs decreases from the peak resonance to $10 \mathrm{~Hz}$, the variability of the inverse GTFs which are applied to the brachial pulse wave increases at this frequency band. The reason might be that the stiffness of blood vessel varies among subjects, and the forward wave meets the reflected wave at different times in a cardiac cycle, leading to the difference among subjects in the shape of the pulse wave and also in the locations of inflection point and notch point in both time and amplitude axis.

Both $H_{g 1}$ and $H_{g 2}$ at $0 \mathrm{~Hz}$ do not equal 1 . One reason for $H_{g 1}$ at $0 \mathrm{~Hz}$ not equaling 1 is that the means of the brachial and aortic pulse waves based on which $H_{g 1}$ was derived are not rigorously equal. Another reason for either $H_{g 1}$ or $H_{g 2}$ at $0 \mathrm{~Hz}$ not equaling 1 might be that the ARX model is an approximation of the relation between brachial and aortic pulse waves. In addition, another reason might be the use of the least squares method to calculate the coefficients of the ARX model.

The prediction errors of BPs are acceptable (SBP error, $0.2 \pm 3.1 \mathrm{mmHg}$; PP error, $-0.9 \pm 3.1 \mathrm{mmHg}$ ). In addition, PP error correlates well with SBP error $(r=0.83, p<0.0001)$, which means that the accuracy of PP estimation is greatly dependent on the accuracy of SBP estimation. This corresponds well with the fact that DBP error is small $(1.1 \pm 1.8 \mathrm{mmHg})$. Large SBP and PP errors might be caused by the inter-patient variance in some frequency components.

The predictions of AI and $p c t P_{n}$ show poor performance (AI percentage error, $-2.1 \pm 32.7 \%$; percentage error of $p c t P_{n}$, $12.4 \pm 9.2 \%)$. The main reason might be that accurate calculation of AI is more dependent on the high-frequency content ( $\geq 8$ harmonics) [9] and the inter-patient variability of the TF appears to be larger at those frequency bands than at lower frequencies (as shown in Fig. 5). The peak resonance frequency of TFs varies within the range of much lower than 8 
TABLE II

PERFORMANCE COMPARISON BETWEEN THE METHODS USED IN THIS PAPER AND By OTHERS

\begin{tabular}{|c|c|c|c|c|c|}
\hline Parameters & Chen [9] & Fetics [11] & Im [23] & NPMA method [25] & This paper \\
\hline SBP error, $\mathrm{mmHg}$ & $0.0 \pm 3.7$ & $0.4 \pm 2.9$ & $-4.8 \pm 3.9$ & $2.1 \pm 3.7$ & $0.2 \pm 3.1$ \\
\hline AI percentage error, $\%$ & $-30 \pm 45$ & $-54 \pm 232$ & $13 \pm 27$ & - & $-2.1 \pm 32.7$ \\
\hline Percentage error of $p c t P n, \%$ & {[} & {[} & 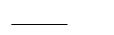 & {[} & $12.4 \pm 9.2$ \\
\hline
\end{tabular}

Hz. Thus, the adjustment of TFs can hardly account for the inter-variability of TFs over $8 \mathrm{~Hz}$. And another reason for the inaccurate prediction of $\mathrm{AI}$ is that the inflection point occurs in the sharp upstroke or down-stroke of the aortic pulse wave and is sometimes nearly invisible, which leads to AI calculation error in both estimated and measured aortic pulse waves. The prediction of ED is acceptable (percentage error, $-2.4 \pm 3.3 \%$ ), which is because that ED is a parameter in time axis and is thus less affected by the sharp upstroke or down-stroke.

In clinical use, calibration error is a common problem when estimating central aortic pulse wave from the peripheral one. Our method does not suffer the pressure difference between the peripheral artery site employed and the artery where the peripheral BP is measured. However, our method is still affected by the error of BP measurement. Future work is needed to improve the accuracy of non-invasive BP measurement.

The ATF method can reliably and accurately estimate the aortic pulse wave using the brachial pulse wave as well as some parameters like SBP, DBP, PP, and ED. However, more future work should be done to improve the accuracy of high-frequency part and its related parameters like AI. Subject-specific physical model, with easily acquiring parameters, is maybe optional.

\section{REFERENCES}

[1] J. Hashimoto, "Central hemodynamics and target organ damage in hypertension," Tohoku J. Exp. Med., vol. 233, no. 1, pp. 1-8, Apr. 2014.

[2] C. Vlachopoulos, K. Aznaouridis, M. F. O'Rourke, M. E. Safar, K. Baou, and C. Stefanadis, "Prediction of cardiovascular events and all-cause mortality with central haemodynamics: a systematic review and meta-analysis," Eur. Heart J., vol. 31, no. 15, pp. 1865-1871, Aug. 2010.

[3] K. L. Wang, H. M. Cheng, S. Y. Chuang, H. A. Spurgeon, C. T. Ting, E. G. Lakatta, F. C. Yin, P. Chou, and C. H. Chen, "Central or peripheral systolic or pulse pressure: which best relates to target organs and future mortality?" J. Hypertens., vol. 27, no. 3, pp. 461-467, Mar. 2009.

[4] R. Pini, M. C. Cavallini, V. Palmieri, N. Marchionni, M. Di Bari, R. B. Devereux, G. Masotti, and M. J. Roman, "Central but not brachial blood pressure predicts cardiovascular events in an unselected geriatric population: the ICARe Dicomano Study," J. Am. Coll. Cardiol., vol. 51, no. 25, pp. 2432-2439, Jun. 2008.

[5] G. F. Mitchell, S. J. Hwang, R. S. Vasan, M. G. Larson, M. J. Pencina, N. M. Hamburg, J. A. Vita, D. Levy, and E. J. Benjamin, "Arterial stiffness and cardiovascular events: the Framingham Heart Study," Circulation, vol. 121, no. 4, pp. 505-511, Feb. 2010.

[6] P. Segers, D. Mahieu, J. Kips, E. Rietzschel, M. De Buyzere, D. De Bacqure, S. Bekaert, G. De Backer, T. Gillebert, P. Verdonck, and L. Van Bortel, "Amplification of the pressure pulse in the upper limb in healthy, middle-aged men and women," Hypertension, vol. 54, no. 2, pp. 414-420, Aug. 2009.

[7] R. Kelly, C. Hayward, A. Avolio, and M. O'Rourke, "Noninvasive determination of age-related changes in the human arterial pulse," Circulation, vol. 80, no. 6, pp. 1652-9, 1998.

[8] K. Takazawa, N. Tanaka, M. Fujita, O. Matsuoka, T. Saiki, M. Aikawa, S. Tamura, and C. Ibukiyama, "Assessment of vasoactive agents and vascular aging by the second derivative of photoplethysmogram waveform," Hypertension, vol. 32, no. 2, pp. 365-370, 1998.

[9] C. H. Chen, E. Nevo, B. Fetics, P. H. Pak, F. C. P. Yin, W. L. Maughan, and D. A. Kass, "Estimation of central aortic pressure waveform by mathematical transformation of radial tonometry pressure validation of generalized transfer function," Circulation, vol. 95, pp. 1827-1836, 1997.

[10] A. L. Pauca, M. F. O'Rourke, and N. D. Kon, "Prospective evaluation of a method for estimating ascending aortic pressure from the radial artery pressure waveform," Hypertension, vol. 38, no. 4, pp. 932-937, Oct. 2001

[11] B. Fetics, E. Nevo, C. H. Chen, and D. A. Kass, "Parametric model derivation of transfer function for noninvasive estimation of aortic pressure by radial tonometry," IEEE Trans. Biomed. Eng., vol. 46, no. 6, pp. 698-706, Jun. 1999.

[12] W. J. Stok, B. E. Westerhof, I. Guelen, and J. M. Karemaker, "Aortic pressure wave reconstruction during exercise is improved by adaptive filtering: a pilot study," Med. Biol. Eng. Comput., vol. 49, no. 8, pp. 909-916, Aug. 2011.

[13] J. O. Hahn, "Individualized estimation of the central aortic blood pressure waveform: a comparative study," IEEE J. Biomed. Health Inform., vol. 18, no. 1, pp. 215-221, Jan. 2014.

[14] J. O. Hahn, A. T. Reisner, F. A. Jaffer, and H. H. Asada, "Subject-specific estimation of central aortic blood pressure using an individualized transfer function: a preliminary feasibility study," IEEE Trans. Inf. Technol. Biomed., vol. 16, no. 2, pp. 212-220, Mar. 2012.

[15] A. Lowe, W. Harrison, E. EI-Aklouk, P. Ruygrok, and A. M. AI-Jumaily, "Non-invasive model-based estimation of aortic pulse pressure using supra-systolic brachial pressure waveforms," J. Biomech., vol. 42, no. 13, pp. 2111-2115, Sep. 2009.

[16] G. Swamy, D. Xu, N. B. Olivier, and R. Mukkamala, "An adaptive transfer function for deriving the aortic pressure waveform from a peripheral artery pressure waveform," Am. J. Physiol., vol. 297, no. 5, pp. H1956-H1963, Nov. 2009.

[17] W. W. Nichols, M. F. O'Rourke, and C. Vlachopoulos, "Pressure pulse waveform analysis," in McDonald's Blood Flow in Arteries: Theoretical, Experimental and Clinical Principles, 6th ed., Ed. London: Arnold, 2011, pp. 595-638.

[18] L. S. Xu, D. Zhang, and K. Q. Wang, "Wavelet-based cascaded adaptive filter for removing baseline drift in pulse waveforms," IEEE Trans. Biomed. Eng., vol. 52, no.11, pp. 1973-1975, Nov. 2005.

[19] L. Ljung, System Identification: Theory for the User (2nd Edition). Ed. New Jersey: Prentice Hall, 1999.

[20] S. M. Romano, and M. Pistolesi, "Assessment of cardiac output from systemic arterial pressure in humans," Crit. Care Med., vol. 30, no. 8, pp. 1834-41, Aug. 2002.

[21] S. Romagnoli, S. Bevilacqua, C. Lazzeri, F. Ciappi, D. Dini, C. Pratesi, G. F. Gensini, and SM. Romano, "Most Care ${ }^{\circledR}$ : a minimally invasive system for hemodynamic monitoring powered by the Pressure Recording Analytical Method (PRAM)," HSR Proc. Intensive Care Cardiovasc. Anesth., vol. 1, no. 2, pp. 20-7, 2009.

[22] A. Donati, A. Carsetti, S. Tondi, C. Scorcella, R. Domizi, E. Damiani, V. Gabbanelli, C. Münch, E. Adrario, P. Pelaia, and M. Cecconi, "Thermodilution vs pressure recording analytical method in hemodynamic stabilized patients," J. Crit. Care., vol. 29, no. 2, pp. 260-4, Apr. 2014.

[23] J. J. Im, R. Wei, Y. C. Lim, C. S. Hwang, H. S. Kim, and M. Y. Rhee, "Indirect measurement of central aortic pressure using carotid and radial pulses," International Journal of Bio-Science \& Bio-Technology, vol. 5, no. 3, pp. 29-39, Jun. 2013.

[24] B. Williams, P. S. Lacy, P. Yan, C. N. Hwee, C. Liang, and C. M. Ting, "Development and validation of a novel method to derive central aortic systolic pressure from the radial pressure waveform using an n-point moving average method," J. Am. Coll. Cardiol., vol. 57, no.8, pp. 951-961, Feb. 2011

[25] Y. T. Shih, H. M. Cheng, S. H. Sung, W. C. Hu, and C. H. Chen, "Application of the n-point moving average method for brachial pressure waveform derived estimation for central aortic systolic pressure," Hypertension, vol. 63, no. 4, pp. 865-870, Apr. 2014. 